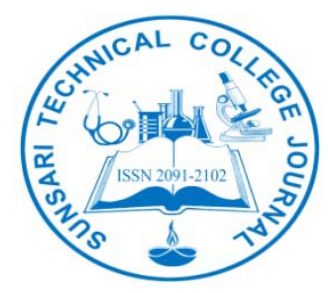

\title{
Editorial
}

\section{COLORANTS IN OUR HEALTH}

DOI: http://dx.doi.org/10.3126/stci.v2i1.14804

"Yellow turmeric, red chilly, green salad and red wine"

Colorants both natural and synthetic have become the integral part of our delicious cuisine. Recently, it had re-emerged with its importance in cosmetics as well as in the treatment of various ailments.

The acceptability and palatability of favoured cuisines and food products has greatly been found to be influenced by the color perception, before its aroma.

The use of cucurmin in its natural form as turmeric powder or haldi for food ingredients and cosmetics has been in practice from time immemorial in Indian culture and tradition and Ayurvedic medicine. Similarly, the representation of red chilly powder (with capsaicin) has been considered as representation of spicy, palatable and inflammatory product and to combat more disturbing pain.

The acceptable daily intake (ADI) of colorant considered safe may not be available for many traditionally used colorant and may not be in practice in many part of the world due to lack of regulatory and monitoring bodies. Therefore, mostly colorant which are considered safe, may infact be harmful to the body causing many adverse health outcome. Although the department of food technology and quality control (DFTQC) is responsible for certifying and monitoring food safely in Nepal (like FDA in the USA and EFSA in Europe), it cannot monitor the complexity of food colorant and nutrient component of all the food items supplied in the market and consumed by the general population. Hence there is a dual challenge of educating the people regarding the used and misuse of colorant and providing service related to food safety and toxicological implication through appropriate analytical laboratories.

The review article on food colorant in this issue of STCJ has elaborately highlighted on the pros and cons of using both natural and synthetic colorant.

Research articles related to such a topic with great health implication must be encouraged. Other articles in this issue of the journal are laying much emphasis in the use of locally available food products and their human consumption and economic implications. Besides, the article in the pharmacologic use of some essential drugs also finds its importance in the journal and hopefully the readers will be benefited from these articles contributed by the local researcher. Albeit in a small scale, it is still a true contribution towards the vast pool of scientific knowledge.

We look forward to receiving a continued support and constructive criticism through feedback for the betterment of the journal.

Dr. Madhab Lamsal, Professor \& Head, Department of Biochemistry, BPKIHS, Dharan, Nepal Chief Editor, STCJ 